\title{
Utilização de um fluxômetro de massa na injeção controlada de gases em vácuo
}

\section{Use of a mass flowmeter in the controlled injection of gases in vacuum}

Pericles Lopes SantAna ${ }^{1, *}$ (D)

1. Universidade Estadual Paulista "Júlio de Mesquita Filho" - Sorocaba (SP), Brazil.

Correspondence author: drsantanapl@gmail.com

Section Editor: Maria Lúcia P. Silva

Received: 20 Jul 2021 Approved: 26 Jan 2022

\begin{abstract}
RESUMO
A medição e o controle de vazão de massa são itens necessários à reprodutibilidade de processos tecnológicos que utilizam gases como matéria-prima, em que a estequiometria e o tempo de processo bem definidos são requeridos. Para medir a vazão de massa, são utilizados os mass flow meters (MFM). Para controlar a vazão de massa é usado o mass flow controller (MFC), que consiste em uma válvula de controle acoplada a um MFM. Por meio de um aparato simples de medida (o calibrador), são feitas a aferição e calibração dos medidores em regime de vácuo. Neste trabalho, explicam-se o princípio físico de funcionamento, o método de aferição e calibração e as aplicações tecnológicas dos medidores. Para tanto, a injeção controlada de nitrogênio em uma câmara de vácuo foi aferida, resultando em uma função linear da pressão de $\mathrm{N}$ em função da temperatura dentro da câmara para um fluxo de 10 sccm.
\end{abstract}

PALAVRAS-CHAVE: Gases, Vácuo, Medidores.

\begin{abstract}
The measurement and control of mass flow are necessary items for the reproducibility of processes technologies that use gases as raw material, in which stoichiometry and process time well defined are required. To measure the mass flow, mass flow meters (MFM) are used. To control the mass flow, the mass flow controller (MFC) is used, which consists of a control coupled to an MFM. By means of a simple measuring apparatus (the calibrator), the gauging and calibration of meters under vacuum. In this work, the physical principle of operation, the method of gauging and calibration and the technological applications of the meters. Therefore,controlled injection of nitrogen into a vacuum chamber was measured, resulting in a linear function of the pressure of $\mathrm{N}$ as a function of the temperature inside the chamber for a flow of $10 \mathrm{sccm}$.
\end{abstract}

KEYWORDS: Gases, Vacuum, Gauges.

\section{INTRODUÇÃO}

O termo vácuo é geralmente usado para denotar um volume ou região do espaço no qual a pressão está abaixo de 760 torr, isto é, abaixo da pressão atmosférica. De acordo com intervalos de pressão abaixo da pressão atmosférica, podem-se considerar diferentes classificações ou ordem de magnitude do vácuo 1 . A divisão da escala de pressão abaixo da atmosférica é arbitrária e um método conveniente de denotação para diferentes fenômenos físicos que ocorrem no intervalo de pressão especificado para cada categoria². Muitas aplicações industriais de vácuo podem ser também classificadas de acordo com estas categorias: baixo e médio vácuo, alto vácuo e ultraalto vácuo. Para uma escolha adequada da instrumentação utilizada nas instalações de vácuo, é fundamental o conhecimento de conceitos envolvendo o transporte e o bombeamento de gases e vapores. 
Os sistemas de vácuo e de fluxo de gás são amplamente utilizados em muitos campos da modernidade física e tecnologia, como pesquisa espacial, superfície, ciência, aceleradores de partículas, deposição de vapor, processamento de semicondutores, implantação de íons, filme fino, metalização e muitas outras aplicações ${ }^{3}$. Para tanto, este trabalho contribui no entendimento da medição de fluxo de massa em estado gasoso, por meio de conceitos básicos da física e química.

\section{REVISÃO BIBLIOGRÁFICA}

\section{Considerações preliminares}

A medição de vazão inclui, no seu sentido mais amplo, a determinação da quantidade de líquidos, gases e sólidos que passa por certo local na unidade de tempo. Quanto ao tipo de medição de vazão para gases, podem-se destacar a medição de vazão por sensores térmicos e a medição de vazão por pressão diferencial.

Os medidores de fluxo de massa térmica (mass flow meters) são sensores de pressão utilizados tanto em processos industriais como em pesquisas básicas e aplicadas. Esses instrumentos têm um tempo de resposta menor que 2 segundos ${ }^{4}$. As determinações de vazão de gases nesse tipo de medidor, que devem ser feitas periodicamente, são em geral feitas no exterior. Isso demanda um custo apreciável se comparado ao de um equipamento novo.

O processo físico básico de medição de fluxo de gases para esses dispositivos é simples. O seu princípio físico está baseado na transferência de calor por condução e convecção nos gases. Por intermédio da troca de energia térmica entre uma fonte externa de energia, por exemplo, um resistor, e o gás, pode-se determinar a quantidade desse gás que passa por um tubo, uma vez sabida a capacidade térmica do gás em questão.

\section{Teoria física para metrologia de vazão em vácuo}

O objetivo desta seção é apresentar de forma sucinta a equação fundamental para o processo de bombeamento em vácuo, fundamental para a maioria dos processos que ocorrem em metrologia em vácuo. Como exemplo de aplicação direta da equação de Clapeyron-Mendeleev, tem-se a Eq. 1.

$$
\mathrm{P} V=n \mathrm{kT}
$$

O método de expansões estáticas pode ser usado extensamente na metrologia em vácuo, pela equação dos gases perfeitos. Dessa forma, derivamos ambos os membros dessa equação em relação ao tempo (Eqs. 2, 3 e 4):

$$
\begin{gathered}
P V=n k T \\
d / d t(P V)=d / d t(n k T) \\
P d V / d t+V d P / d t=k T d n / d t+k n d T / d t
\end{gathered}
$$

Para a maior parte dos sistemas de vácuo, a temperatura e o volume da câmara de vácuo são mantidos constantes (Eq. 5):

$$
V \mathrm{dP} / \mathrm{dt}=\mathrm{kT} \mathrm{dn} / \mathrm{dt}
$$

Em que: $\mathrm{V}$ = volume da câmara de gases; $\mathrm{P}$ = pressão de gases dentro da câmara; $\mathrm{K}$ = constante de Boltzmann; $\mathrm{T}$ = temperatura dentro da câmara; $\mathrm{n}$ = número de moles ou quantidade de gás dentro do sistema.

Por causa do movimento de translação de átomos e moléculas, temos associado a ele uma energia cinética (Ecm). Há três graus de liberdade no movimento de translação, uma para cada direção possível do movimento. Para cada grau de liberdade, a energia cinética média de translação é 1/2kT, resultado este obtido pelo princípio da equipartição de energia. Portanto, a energia cinética média total de translação por molécula é (Eq. 6): 


$$
\mathrm{Ecm}=3(1 / 2 \mathrm{kT})=3 / 2 \mathrm{kT}
$$

Para N moléculas (Eq. 7)

$$
\mathrm{E}=\mathrm{NEcm}=3 / 2 \mathrm{NkT}
$$

Usando a equação dos gases perfeitos nesse último resultado, tem-se a Eq. 8:

$$
E=3 / 2 N k T=3 / 2 P V
$$

Tomando a derivada em relação ao tempo, associamos a variação de energia cinética média total de translação à variação de pressão (Eq. 9):

$$
d E / d t=d / d t(N E c m)=E c m d N / d t=3 / 2 k T d N / d t=3 / 2 V d P / d t, \text { ou seja, } V d P / d t=2 / 3 d E / d t
$$

Considerando um sistema de vácuo com várias fontes de gases possíveis presentes na câmara de vácuo, definimos a grandeza vazão, Q = dN/dt. Ela expressa a variação do número de moléculas na câmara de vácuo, no tempo. Como P V = n K T, logo (Eqs. 10 e 11):

$$
\begin{gathered}
\mathrm{Q}^{\prime}=\mathrm{dN} / \mathrm{dt}=\mathrm{d} / \mathrm{dt}(\mathrm{P} \vee / \mathrm{kT})=1 / \mathrm{kT} \mathrm{d} / \mathrm{dt}(\mathrm{PV}) \\
\mathrm{Q}^{\prime}=\mathrm{V} / \mathrm{k} T \mathrm{dP} / \mathrm{dt}
\end{gathered}
$$

Como obtido anteriormente, sabe-se que (Eq. 12):

$$
\mathrm{dE} / \mathrm{dt}=3 / 2 \mathrm{kT} d \mathrm{~N} / \mathrm{dt}=3 / 2 \mathrm{~V} d \mathrm{P} / \mathrm{dt}
$$

Logo (Eqs. 13 e 14):

$$
\begin{aligned}
& V \mathrm{dP} / \mathrm{dt}=2 / 3 \mathrm{dE} / \mathrm{dt} \\
& \mathrm{Q}^{\prime}=2 / 31 / \mathrm{kT} \mathrm{dE} / \mathrm{dt}
\end{aligned}
$$

Definindo a grandeza throughput como (Eqs. 15 e 16):

$$
\begin{gathered}
Q=K T Q^{\prime} \\
Q=2 / 3 d E / d t
\end{gathered}
$$

O throughput é igual a 2/3 da variação no tempo da energia cinética média do movimento de translação das moléculas na câmara de vácuo ou (Eq. 17):

$$
Q=K T d N / d t
$$

Para determinação da condutância e velocidade de bombardeamento, o princípio está baseado também na medição da variação de pressão no tempo.

\section{Princípio físico do instrumento}

O princípio físico de funcionamento de um mass flow meter (MFM) é o mesmo da torneira elétrica. Baseia-se no princípio da conservação de energia, em que a potência dissipada por um resistor é transferida ao líquido que passa pela torneira, uma vez sabido o calor específico desse líquido 4 . A Fig. 1 ilustra a torneira elétrica 


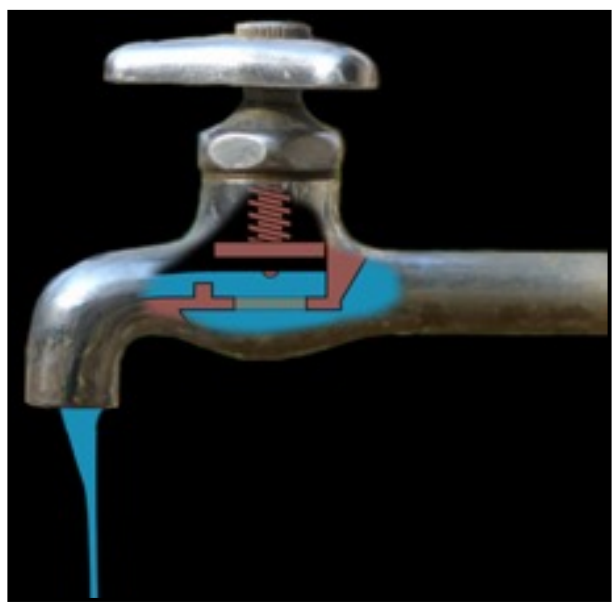

Figura 1: Torneira elétrica.

O simples entendimento desse dispositivo pode ajudar bastante no entendimento de um MFM. Com uma diferença de temperatura existente entre dois terminais ou dois pontos de um resistor, admite-se por conservação de energia que, sob uma diferença de potencial elétrica, a potência dissipada por esse resistor é absorvida pelo fluido que atravessa esse mesmo resistor. Portanto, pelo princípio da conservação de energia, pode-se conhecer a quantidade de fluido que passa pela torneira em um intervalo de tempo, isto é (Eq. 18):

$$
P(t)=V i
$$

Sendo P a potência dissipada pelo resistor.

Deve-se descobrir $\Delta \mathrm{m} / \Delta \mathrm{t}$ (Eqs. 19 e 20):

$$
\begin{aligned}
V i \Delta t & =\Delta m c \Delta T \\
\Delta m / \Delta t & =V i / c \Delta T
\end{aligned}
$$

Em que: c = calor específico da água.

Na Fig. 2, uma ilustração desenvolvida por um fabricante desses dispositivos, pode-se visualizar o instrumento em seu interior.

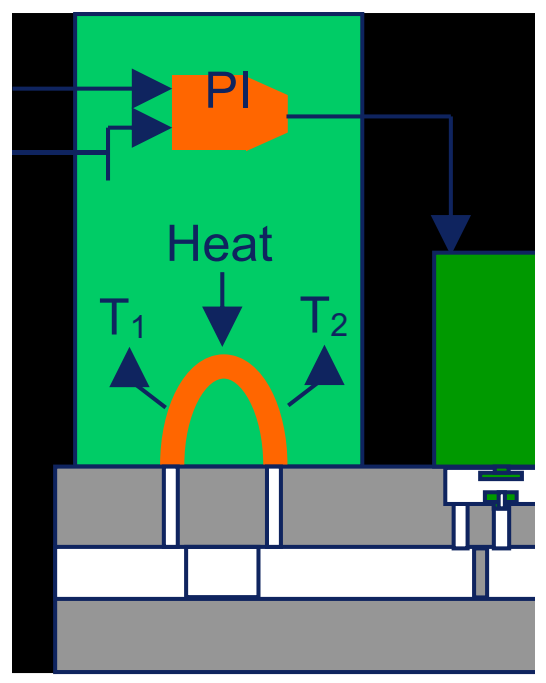

Figura 2: Mass flow controller (Modelo da empresa Bronkhorst.) 


\section{Termistores}

Termistores são semicondutores sensíveis à temperatura. Três tipos de termistores cerâmicos são muito utilizados: termistores com coeficiente de temperatura positivo (PTC), termistores com coeficiente de temperatura negativo (NTC), e termistores de temperaturas críticas.

Termistores NTC são utilizados como fluxômetro, para os quais a velocidade de fluxo é medida por monitoramento de diferentes temperaturas entre os dois termistores. Um aquecedor posicionado entre os dois termistores é utilizado como controlador de fluxo para proteger diodos, fusíveis, interruptores e lâmpadas elétricas.

À medida que a diferença entre as temperaturas T1 e T2 aumenta, a resistividade elétrica diminui, de modo que um sinal de corrente pode ser observado e enviado em forma de output (sinal de saída), que pode ser medido em amperes ou volts. Nesse tipo de instrumento, o sinal pode variar de 0 a 5 volts VDC.

A termorresistência PT-100 de platina é a mais usada industrialmente em função de sua grande estabilidade, larga faixa de utilização (de -270 a $660^{\circ} \mathrm{C}$ ) e alta precisão. Já a estabilidade diz respeito às capacidades do sensor de manter e reproduzir suas características na faixa especificada de operação.

Outro fator importante é a repetibilidade, que é a característica de confiabilidade da termorresistência. A repetibilidade deve ser medida com leitura de temperaturas consecutivas verificando-se a variação encontrada quando a medição é feita novamente às mesmas temperaturas. O tempo de resposta é importante em aplicações em que a temperatura do meio em que se realiza a medição está sujeito a mudanças bruscas.

\section{Gas correlation factor}

O gas correlation factor (GCF) é usado para indicar a proporção das taxas de fluxo de diferentes gases que vão produzir a mesma voltagem de saída indicada pelo sensor. Esse fator é uma função do calor específico, da densidade e da estrutura molecular dos gases. O nitrogênio é usado como referência para esse fator (Eq. 21).

$$
\text { GCFx }=0,3106 \times S / d x \times C p x
$$

GCFx = fator de correção para o gás x; 0,3106 = número que depende da densidade e do calor específico do nitrogênio; $\mathrm{S}$ = fator de correção da estrutura molecular; 1,030 = gás monoatômico; 1,000 = gás diatômico; 0,941 = gás triatômico; 0,880 = gás poliatômico; $\mathrm{dx}=$ densidade padrão do gás $\times$ em g/L a $0^{\circ} \mathrm{C}$ e $1 \mathrm{~atm} ; \mathrm{Cpx}=$ calor específico de $\times$ em cal/g ${ }^{\circ} \mathrm{C}$

Para uma mistura de gases (Eq. 22):

$$
\mathrm{GCFm}=0,3106(\mathrm{a} 1 \mathrm{~s} 1+\mathrm{a} 2 \mathrm{~s} 2+\ldots+\mathrm{ansn}) /(\mathrm{a} 1 \mathrm{~d} 1 \mathrm{Cp} 1+\mathrm{a} 2 \mathrm{~d} 2 \mathrm{Cp} 2+\ldots+\mathrm{andnCpn})
$$

Em que: ai = a fração de gases

\section{Experimental}

O sistema experimental consiste em duas câmaras cilíndricas de aço inoxidável conectadas por um tubo de aço inoxidável, com entrada para o gás nitrogênio, como visto na Figura 3.

Entre duas câmaras, foram posicionados dois medidores de pressão: um medidor de pressão, por meio de um manômetro de mercúrio, e um fluxômetro de massa, mediante o fluxo de 10 sccm à pressão de 0.1 Pa.

\section{RESULTADOS}

Para cada valor de pressão, foram coletados valores de temperatura que aumentam linearmente com o tempo. A equação derivada da equação de Clapeyron corresponde ao gráfico da Fig. 4. Matematicamente a equação para vazão pode ser escrita como a Eq. 23:

$$
\mathrm{Q}=\mathrm{V} \Delta \mathrm{P}(\mathrm{t}) / \Delta \mathrm{t}
$$




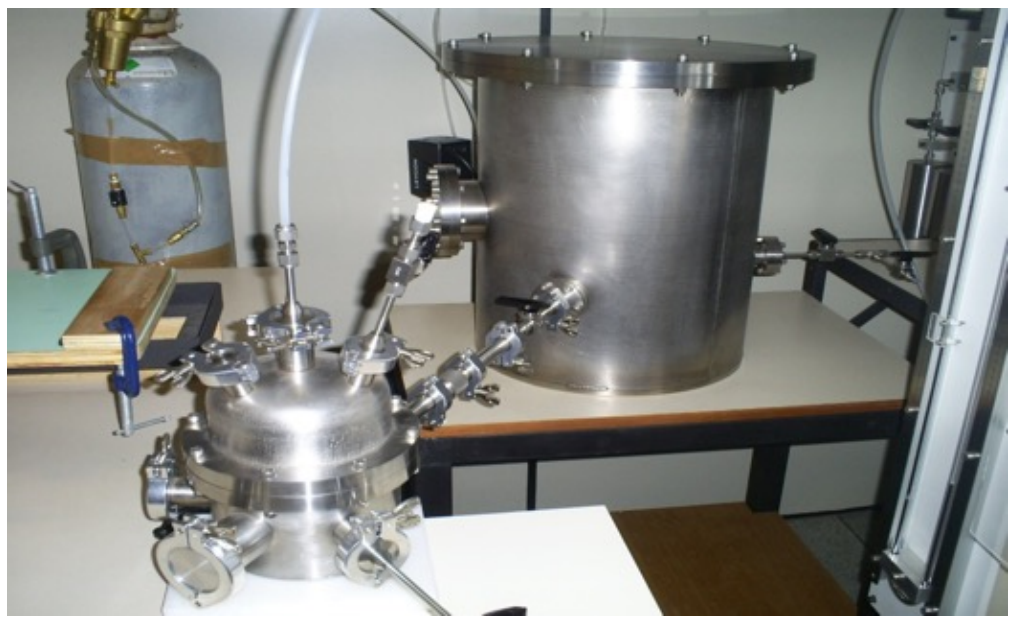

Figura 3: Aparato experimental: duas câmaras de vácuo cilíndricas acopladas a um medidor de fluxo, um manômetro de tubo em U e uma entrada para gás nitrogênio.

Medidas de Vazão

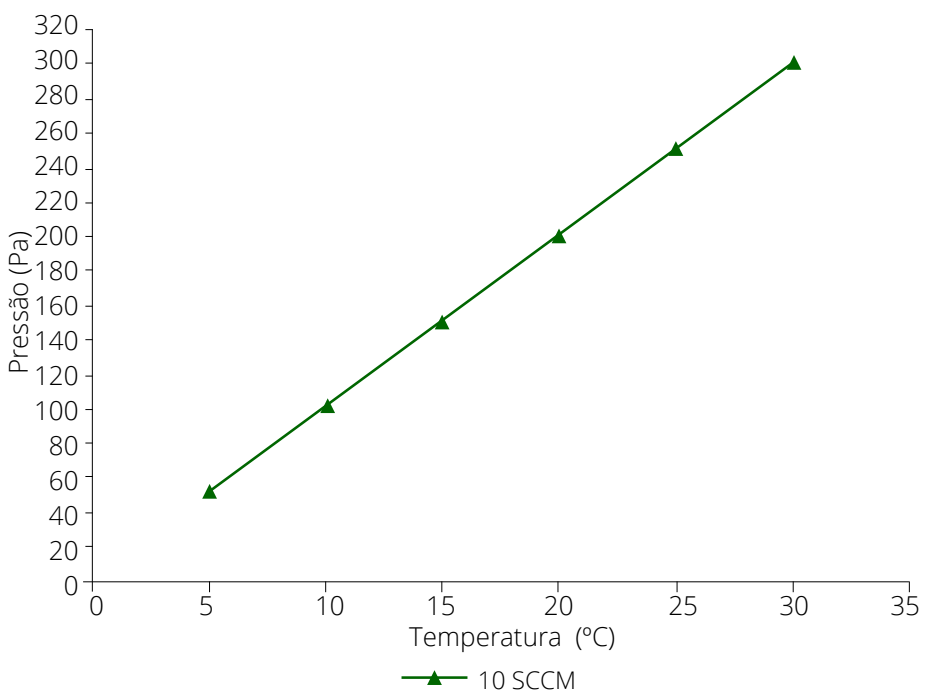

Figura 4: Variação de pressão na câmara de descarga em função da temperatura para o fluxo de 10 sccm.

\section{CONCLUSÕES}

Esses tipos de dispositivos são fortemente utilizados na indústria de semicondutores para diversas aplicações em microeletrônica, na indústria petroquímica, em laboratórios de tecnologia a vácuo, nas indústrias alimentícia e de embalagens etc. Existe atualmente um campo de aplicação muito grande, em que a instrumentação, a medição e o controle se tornam fundamentais à obtenção de produtos com alta qualidade e nível tecnológico. Neste trabalho foi demonstrado de que forma se pode utilizar um fluxômetro de vácuo na injeção controlada de gases usando gás nitrogênio. Na determinação da vazão, foi plotada a reta resultante de pressão (50 a 300 Pa) em função da temperatura $\left(5 \mathrm{a} 30^{\circ} \mathrm{C}\right.$ ) para o fluxo de $10 \mathrm{sccm}$.

\section{FINANCIAMENTO}

Não se aplica. 


\section{DISPONIBILIDADE DE DADOS DE PESQUISA}

Todos os dados foram gerados/analisados no presente estudo.

\section{AGRADECIMENTOS}

Ao professor doutor Francisco Tadeu Degasperi, da Faculdade de Tecnologia de São Paulo (Fatec-SP).

\section{REFERÊNCIAS}

1. MKS. MKS Corporate Social Responsibility Report [Internet]. [acessado em julho 2021]. Disponível em: http:// www.mks.com

2. Degasperi FT. Modelagem e análise detalhada de sistemas de vácuo [dissertação de mestrado]. Campinas: Unicamp; 2002.

3. Chambers A, Fitch W, Coldfield S. Basic vacuum technology. 2ª ed.: Taylor \& Francis Group; 1998.

4. Degasperi FT. Metrologia de pressão e vazão em tecnologia de vácuo. São Paulo: Fatec; 2006.

5. Manual MKS tipos 1179 A e 2179 A Mass Flow Controller. https://www.mksinst.com/resourceListing/manuals

6. Hoffman D, Singh B, Thomas III JH. Handbook of vacuum science and technology. San Diego:1ª Ed.; 1998. 